\title{
Editorial: Controle por pares e a função do revisor - premissas e orientações para uma avaliação consistente
}

\author{
Editorial: Peer review and the reviewer's role - principles and \\ alignments to an effective review
}

\section{Vinicius Gomes de Vasconcellos}

Doutorando em Direito na Universidade de São Paulo - São Paulo/SP Editor-chefe da RBDPP e editor-assistente da RBCCRIM vgomesv@gmail.com

lattes.cnpq.br/9628659956663949

D. orcid.org/0000-0003-2020-5516

p publons.com/a/1174099/

Continuando com a análise das fases e dos papeis dos atores do processo editorial científico, ${ }^{1}$ após assentar as premissas sobre o periódico científico e a atuação do editor, ${ }^{2}$ este editorial pretende explorar as características fundamentais do "controle por pares" e, consequentemente, do revisor na produção do conhecimento em revistas científicas. Trata-se, certamente, do núcleo do processo editorial científico como concebido contemporaneamente, ${ }^{3}$ tendo em vista que a ampla maioria dos periódicos adota o sistema de controle por pares para seleção de artigos, o que é, inclusive, imposto pelos órgãos de certificação, como a Comissão Qualis/CAPES.

1 Assumindo-se os potenciais problemas do sistema de controle por pares, mas ressaltando suas possíveis contribuições, deve-se adotar postura crítica, de modo que “(...) uma compreensão clara de cada uma das funções desenvolvidas por cada um dos atores contribui para minimizar, ainda que não elimine, os potenciais efeitos negativos sobre a publicação dos melhores trabalhos em qualquer área da ciência" (MARTÍNEZ. La revisión por pares y la selección de artículos para publicación, p. 30) (tradução livre).

2 VASCONCELLOS. Editorial: a função do periódico científico e do editor para a produção do conhecimento no Direito e nas ciências criminais, p. 9-17.

3 BENOS; KIRK; HALL. How to review a paper, p. 50. 
Contudo, tal temática é pouco explorada cientificamente no meio acadêmico brasileiro e, ainda menos, no Direito, o que prejudica a preparação de bons avaliadores e a qualidade do controle por pares. Desse modo, na melhor das hipóteses, essa tarefa acaba sendo aprendida exclusivamente pela experiência individual, em lugar de desenvolvida por orientações consolidadas e fundamentadas. Sintoma desse quadro é a raridade (ou inexistência) de disciplinas ou seminários nos PPGs brasileiros que preparem o acadêmico para a sua atuação como revisor de periódicos, ${ }^{4}$ ignorando-se que isso será indispensável para a manutenção do sistema de produção de conhecimento científico: cada vez mais se cobra produtividade em termos de publicação de artigos em periódicos, mas, inconsequentemente, pouco (ou quase nada) se prepara, incentiva ou valoriza a atuação de editores e avaliadores. ${ }^{5}$

Assim, este editorial aspira analisar a posição e a função do revisor no processo editorial científico para, então, assentar certas premissas importantes para sua atuação e indicar algumas orientações com o objetivo de fomentar o aprimoramento das avaliações realizadas. Para tanto, utilizar-se-á referencial bibliográfico específico e embasado em pesquisas empíricas sempre que possível, mas também percepções de experiência como autor, revisor e editor. Nesse sentido, o texto se estruturará em três partes: (I) definição e características do controle por pares; (II) premissas de atuação do revisor; e, (III) orientações para redação de pareceres consistentes.

Inicialmente (I), deve-se assentar que o controle por pares é um mecanismo de avaliação de trabalhos científicos utilizado em diversos

4 FERREIRA; PINTO; BELFORT. O que é uma boa revisão de artigo em Administração? p. 92. Assim também no exterior: ROBERTS; COVERDALE; EDENHARDER; LOUIE. How to Review a Manuscript, p. 81.

5 Considerando a crescente cobrança imposta para que os pesquisadores publiquem artigos em periódicos científicos e a necessidade de respeito aos parâmetros básicos do processo editorial, se a CAPES e o próprio meio acadêmico não melhor valorarem a atuação dos acadêmicos como pareceristas e editores, o sistema logo entrará em colapso por falta de pareceres suficientes para a avaliação dos trabalhos submetidos. Sobre a relação entre revisão por pares e a cultura de produtivismo acadêmico, ver: SHIGAKI; PATRUS. Revisão por pares e produtivismo acadêmico sob a ótica de avaliadores de artigos de periódicos em Administração, p. 402-407. 
meios, não só em periódicos científicos, mas também nos próprios programas de pós-graduação (bancas de mestrado, doutorado, etc.) e em análises para concessões de financiamentos por órgãos de fomento, por exemplo. Trata-se de "um processo baseado na premissa de que os revisores são indivíduos competentes na área do artigo que avaliam, que são independentes, objetivos e que dedicam seu melhor esforço à avaliação". ${ }^{6}$ Ou seja, parte-se da ideia de que os pares (especialistas na mesma área) dos próprios autores dos artigos têm competências científicas e técnicas para analisar criticamente os elementos formais e substanciais das pesquisas que se pretende divulgar ao meio acadêmico e à sociedade. ${ }^{7}$ Assim, assegura-se à publicação um "selo de certificação, sinalizando que o trabalho foi escrutinado pelos pares". ${ }^{8}$

Nas revistas científicas, o revisor assume duas funções primordiais no processo editorial: 1) auxiliar e orientar o editor na avaliação e seleção dos trabalhos submetidos ao periódico (gatekeeping role); e, 2) ajudar o autor a melhorar o trabalho com críticas e sugestões construtivas. ${ }^{9} \mathrm{Ou}$ seja, como primeira constatação que deve ser considerada, afirma-se que o parecer deve ser redigido considerando seus destinatários: editor e autor. ${ }^{10}$

Em termos gerais, o controle por pares pode ser realizado de três modos distintos, em relação ao anonimato dos sujeitos envolvidos. ${ }^{11}$ O sistema majoritariamente utilizado ${ }^{12}$ (e imposto pela Qualis-Direito) ${ }^{13}$

6 FERREIRA; CANELA; PINTO. O processo editorial nos periódicos e sugestões para a publicação, p. 5.

7 SPYNS; VIDAL. Scientific Peer Reviewing, p. 2; WERLANG. Revisão por pares, p. 29.

8 FERREIRA; PINTO; BELFORT. O que é uma boa revisão de artigo em Administração? p. 89.

9 FERREIRA. Como rever um artigo, p. 1; ROBERTS; COVERDALE; EDENHARDER; LOUIE. How to Review a Manuscript, p. 81; PROVENZALE; STANLEY. A systematic guide to reviewing a manuscript, p. 848.

10 PLATA-CAVIDES; CÓRDOBA-SALGADO; TRZESNIAK. Dictámenes en Revistas Científicas, p. 51; LEUNG; LAW; KUCUKUSTA; GUILLET. How to review journal manuscripts, p. 46.

11 ALI; WATSON. Peer review and the publication process, p. 195-197.

12 WERLANG. Revisão por pares, p. 74.

13 Relatório com os critérios adotados em 2016 para a classificação Qualis-Direito disponível em: <http://www.capes.gov.br/images/documentos/Qualis_periodicos_2016/Direito_Qualis_.pdf>. Acesso em: 29 mar. 2017. 
é o duplo-cego (double-blind), em que tanto os avaliadores não têm acesso aos dados do autor da submissão, como o autor não saberá quem revisou seu texto, de modo que somente o editor tem conhecimento das identidades para realizar o manejo das comunicações. Já no sistema de cego-simples (single-blind), autores não sabem quem foram os avaliadores, mas estes recebem os artigos com identificação do pesquisador. Por fim, no sistema aberto (open review) todos os envolvidos são mutuamente identificados e, em algumas revistas, os pareceres são, inclusive, publicados juntamente com o artigo aprovado.

O sistema duplo-cego possui os benefícios de tentar reduzir atuações parciais de avaliadores em razão de predisposições em relação a autores específicos, ${ }^{14}$ além de possibilitar aos pareceristas críticas profundas sem preocupação de retaliações posteriores, sendo por isso apontado como preferido por parte majoritária dos avaliadores. ${ }^{15}$ Contudo, esse anonimato também possibilita abusos, como pareceres agressivos ou inconsistentes (o que deve ser controlado e repudiado pelo editor), além de fomentar revisões não tão dedicadas em razão do pouco reconhecimento do trabalho realizado. ${ }^{16}$ Por isso, a adoção do sistema aberto é uma tendência com o objetivo de valorizar e responsabilizar o avaliador pelos pareceres realizados, caracterizando uma opção interessante a ser considerada pelos periódicos jurídicos brasileiros futuramente. ${ }^{17}$

Com a pretensão de assegurar avaliações imparciais e sem receios de represálias, a RBDPP atualmente adota o sistema duplo-cego, mantendo o anonimato recíproco entre autores e avaliadores durante

14 Contudo, na prática, assegurar totalmente o anonimato dos autores é muito complicado (ou até impossível em alguns casos), pois, sendo o avaliador um especialista na área temática e, em regra, os pesquisadores de cada campo conhecem os estilos e temas pesquisados reciprocamente, presumir a autoria do artigo (mesmo sem qualquer informação de identificação) é relativamente possível, de modo que verificações de conflito de interesses são importantes ainda que em periódicos com avaliação dupla-cega.

15 LEUNG; LAW; KUCUKUSTA; GUILLET. How to review journal manuscripts, p. 51.

16 MARTÍNEZ. La revisión por pares y la selección de artículos para publicación, p. 28-30.

17 Afirmando que a abertura/publicidade no processo editorial é fundamental para o "controle sobre o controle por pares": COMMONER. Peering at Peer Review, p. 29. 
o processo editorial. Contudo, em prol da valorização da atuação do revisor, permite-se a posterior publicação do parecer em sistemas de certificação (como Publons) ${ }^{18}$ ou na própria revista, na seção de Críticas Científicas. Ou seja, o anonimato é uma prerrogativa do avaliador, que pode ser por ele renunciada para reconhecimento do seu trabalho após o encerramento do processo editorial do respectivo artigo.

Atualmente afirma-se que a maioria dos pesquisadores atua como parecerista de periódicos por quatro motivos: "fazer a sua parte na comunidade acadêmica", "apreciar ajudar no aprimoramento dos artigos", "gostar de ter contato com os estudos antes de sua publicação" (ou seja, conhecer o que está sendo produzido nas pesquisas de ponta antes da divulgação ampla) e "retribuir as avaliações feitas por outros pareceristas aos próprios trabalhos". ${ }^{19}$ Resta evidente que qualquer pesquisador inserido no meio acadêmico deve colaborar com o processo editorial dos periódicos científicos, pois todos se utilizam desse sistema ao submeterem seus próprios artigos e aguardarem por avaliações consistentes e em prazo razoável. Ou seja, a atuação como revisor de periódicos precisa ser um elemento fundamental e constante na carreira acadêmica de qualquer pesquisador. ${ }^{20}$ Além disso, o avalia-

18 A RBDPP firmou pareceria com o inovador sistema Publons, um mecanismo de certificação e reconhecimento da produção técnica desenvolvida pelo revisor nas colaborações realizadas ao avaliar os artigos submetidos. Após o envio do parecer, o avaliador poderá inserir tal informação em seu perfil Publons (pelo próprio sistema da RBDPP ou por e-mail encaminhado após a confirmação da avaliação), o que garante a confiabilidade da informação, pois o sistema possui mecanismo de checagem da atividade descrita. Sobre isso, ver: RAJPERT-DE MEYTS; LOSITO; CARRELL. Rewarding peer-review work, p. 985-986; SMITH. Will Publons Popularize the Scientific PeerReview Process?, p. 265-266.

19 WARE. Peer review, p. 8 (tradução livre). Assim também em pesquisa mais recente: WARE. Peer review survey 2015, p. 36. Em estudo com avaliadores brasileiros: SHIGAKI; PATRUS. Revisão por pares e produtivismo acadêmico sob a ótica de avaliadores de artigos de periódicos em Administração, p. 410-413.

20 “A revisão por pares é essencial para se produzir ciência de qualidade. Revisar artigo é atividade científica. A reputação de um pesquisador se constrói não só pela qualidade dos seus artigos, mas também pela qualidade da sua revisão nos textos dos pares. No sistema de valores da comunidade científica, a recusa sistemática de revisões pelo pesquisador é negativa" (DESLANDES; SILVA. Revisão por pares, p. 421). 
dor desenvolve suas próprias habilidades como cientista e escritor ao ler e revisar trabalhos científicos, além de ao comparar seus pareceres com os demais envolvidos no processo editorial do artigo com o feedback do editor. ${ }^{21}$

Certamente, o processo editorial científico pautado pelo controle por pares não é um mecanismo perfeito e precisa ser analisado criticamente, com o objetivo de reduzir possíveis espaços de arbitrariedade e erro. ${ }^{22}$ Conforme Shigaki e Patrus, "as críticas mais comuns, aqui elencadas em ordem decrescente de citações, estão relacionadas a tempo, sobrecarga de trabalho, subjetividade das avaliações, falta de reconhecimento do trabalho do avaliador, falta de padrão de qualidade na seleção dos avaliadores, necessidade de ter mais avaliadores para criar rotatividade entre eles, parcialidade dos editores e abuso de poder". ${ }^{23}$

Algo facilmente perceptível por autores e editores, que em uma primeira análise seria um sinal da fragilidade do processo de avaliação, é a alta frequência de discordâncias entre os pareceres emitidos a um artigo: raro é o caso em que dois avaliadores fazem críticas semelhantes e apontam os mesmos problemas no texto. ${ }^{24}$ Aqui se destaca a importância da atuação do editor que, a partir da leitura do artigo e dos pareceres, de-

21 SPYNS; VIDAL. Scientific Peer Reviewing, p. 2.

22 Estudos demonstram que alguns dos artigos mais relevantes em suas áreas foram inicialmente rejeitados por periódicos, o que impõe a problematização da ideia de que o controle por pares é um meio perfeito para seleção dos melhores trabalhos científicos. Sobre isso, ver: CAMPANARIO, Have Referees Rejected Some of the Most-Cited Articles of All Times? p. 302-310; CAMPANARIO. Peer review for journals as it stands today, p. 198-199. Em relação à (não) absoluta fiabilidade de artigos publicados após controle por pares e os problemas da utilização de tal conhecimento como prova científica em processos judiciais, ver: HAACK. La evaluación por pares y la publicación, p. 15-40. Em geral, sobre os problemas do controle por pares: LOCK. A difficult balance, p. 23-55.

SHIGAKI; PATRUS. Revisão por pares e produtivismo acadêmico sob a ótica de avaliadores de artigos de periódicos em Administração, p. 413. Afirmando que a principal crítica é a demora do processo de avaliação com controle por pares: WERLANG. Revisão por pares, p. 75.

FISKE; FOGG. But the reviewers are making different criticisms of my paper, p. 591-598; WARE. Peer review, p. 16; CORONEL; OPTHOF. The role of the reviewer in editorial decision-making, p. 263; CAMPANARIO. Peer review for journals as it stands today, p. 191. 
verá decidir e consolidar as críticas para orientação do autor. ${ }^{25}$ Portanto, esperar-se uniformidade de visões entre avaliadores é inviável, mas é exatamente das divergências que resultarão maiores contribuições para o aprimoramento do texto. Certamente, o risco de abusos por parte do editor, que precisa conhecer os dados dos autores e avaliadores para possibilitar o contato e a estruturação de um fluxo editorial otimizado, é inevitável, mas para reduzi-lo deve-se consolidar ao máximo mecanismos de transparência do processo editorial e de controles internos e externos da sua atuação. ${ }^{26}$

Diante do exposto, inegáveis são os problemas relacionados ao sistema de controle por pares para seleção de trabalhos e, assim, do conhecimento produzido em determinada área do saber, de modo que a constante busca por redução dos riscos de abusos e arbitrariedades deve ser primordial. Contudo, além de ser a única opção viável atualmente, ${ }^{27}$ também é inquestionável que, quando adequadamente realizado, o processo editorial com controle por pares contribui concretamente ao aprimoramento dos artigos e fornece elementos razoáveis para ajudar a equipe editorial do periódico na seleção das submissões. ${ }^{28}$ Nesse sentido, na

25 SOLOMON. The Role of Peer Review for Scholarly Journals in the Information Age, p. 3; FERREIRA; PINTO; BELFORT. O que é uma boa revisão de artigo em Administração? p. 101.

26 WICHERTS. Peer Review Quality and Transparency of the Peer-Review Process in Open Access and Subscription Journals, p. 15-17.

27 Em termos semelhantes: WINCK; WEDZICHA; FONSECA; AZEVEDO. To publish or perish, p. 101; LEE. How to be a great reviewer, p. 158; MARTÍNEZ. La revisión por pares y la selección de artículos para publicación, p. 28; LARSON; CHUNG. A systematic review of peer review for scientific manuscripts, p. 38; BIGGS. The impact of peer review on intellectual freedom, p. 162-163; WERLANG. Revisão por pares, p. 31; HAMES. Peer Review and Manuscript Management in Scientific Journals, p. 3.

28 Em pesquisa empírica que analisou artigos antes e após o processo editorial da revista Annals of Internal Medicine demonstrou-se que, em regra, houve efetivos aprimoramentos em 33 dos 34 critérios examinados: GOODMAN; BERLIN; FLETCHER; FLETCHER. Manuscript quality before and after peer review and editing at Annals of Internal Medicine, p. 11-21. Em recente pesquisa realizada por meio de entrevista a 2004 avaliadores, 74\% dos revisores afirmaram que o controle por pares acarreta efetivo aprimoramento aos artigos científicos e $82 \%$ concordou com a frase "sem revisão por pares não há controle sobre a comunicação científica” (WARE. Peer review survey 2015, p. 10) (tradução livre). De modo semelhante, na pesquisa de 2009: WARE. Peer 
doutrina brasileira, afirma-se que, "apesar de suas limitações, o processo de revisão por pares é imprescindível para a difusão do conhecimento, pois é uma etapa essencial no julgamento de manuscritos científicos", de modo que "é importante que se façam mudanças no sentido de reduzir a subjetividade e, desta forma, garantir credibilidade ao processo". ${ }^{29}$

Partindo-se da conclusão de que o controle por pares é fundamental para a produção do conhecimento científico nas diversas áreas, ressalta-se a relevância da atuação do revisor como ator determinante do processo editorial. Certamente, o aprimoramento desse sistema depende em muito da consolidação da atuação dos pareceristas com a ampliação do percentual de avaliações de excelência. Para tanto, pensa-se que algumas premissas fundamentais (II) devem ser assumidas pelos revisores:

a) O parecer não é um voto e a aprovação/rejeição do artigo não é necessariamente determinada pela orientação indicada pela maioria dos avaliadores. ${ }^{30}$ Ele é uma recomendação, cujo elemento fundamental é a motivação, que deve orientar a decisão tomada pelo editor do melhor modo possível. Por isso, um parecer sem fundamentação, somente com marcações em eventuais questões objetivas, não pode ser considerado pelo editor, sendo, assim, nulo.

O parecer é, primeiramente, um instrumento de convencimento do editor e precisa indicar os motivos que aconselham a aprovação ou a rejeição do artigo. Nesse sentido, é importante esclarecer que a sugestão

review, p. 14. Assim também em relação à satisfação positiva dos autores e editores sobre o controle por pares: SHATTELL; CHINN; THOMAS; COWLING. Authors' and editors' perspectives on peer review quality in three scholarly nursing journals, p. 61. De modo semelhante em pesquisa com acadêmicos de marketing: BAILEY; HAIR; HERMANSON; CRITTENDEN. Marketing Academics' Perceptions of the Peer Review Process, p. 263-278. Assentando que artigos avaliadores por controle cego por pares são mais citados (possuem maior impacto): LABAND; PIETTE. A citation analysis of the impact of blinded peer review, p. 147-149.

JENAL; VITURI; EZAÍAS; SILVA; CALIRI. O processo de revisão por pares, p. 808. Para uma proposta de estabelecimento de critérios para verificação de artigos científicos: CAPPARELLI. Editorial: Sobre a exigência de um método de escrita de artigos científicos e de estudo do Direito Processual Penal, p. 24-25.

30 LEOPOLD. Editorial: Peer Review and the Editorial Process, p. 1-2; TRZESNIAK. A estrutura editorial de um periódico científico, p. 95; HAMES. Peer Review and Manuscript Management in Scientific Journals, p. 4. 
de publicação deve ser tão motivada quanto a de rejeição: ${ }^{31}$ alguns avaliadores pensam que a aprovação não necessita de justificação e isso está equivocado, pois, em uma revista de excelência, somente artigos que efetivamente apresentem qualidades destacadas e contribuições relevantes deverão ser publicados, o que precisa ser indicado pelo avaliador ao editor.

b) O parecer deve colaborar ao concreto aprimoramento do artigo avaliado (orientação construtiva). ${ }^{32}$ Além de dirigido ao editor, o parecer necessita ser concebido como meio de ajuda ao autor, de modo que mais importante do que destruir completamente o texto é sugerir correções e complementações para sua revisão, ou seja, "a função do avaliador não é somente selecionar os artigos que devem ser publicados, mas também fornecer meios para que se aprimore a qualidade do manuscrito". ${ }^{33} \mathrm{Ou}$, em termos mais precisos, o parecerista não deve buscar somente motivos para rejeição, mas, especialmente, ponderar os pontos positivos do artigo e os potenciais meios para aprimoramento de modo a consolidá-lo como uma referência científica consistente. ${ }^{34}$

c) Além de verificar aspectos formais do trabalho, é primordial adentrar às ideias substanciais do artigo, às teses por ele propostas, às premissas teóricas adotadas: certamente, apontar problemas formais é fundamental, mas o parecer deve propiciar uma verificação e um questionamento das ideias sustentadas no trabalho. Portanto, o revisor não deve se limitar a apontar somente deficiências formais e se contentar em sugerir uma rejeição simplesmente por tais motivos (especialmente se tais problemas forem sanáveis em curto prazo), pois, como especialista na temática do artigo, o avaliador deve ajudar o editor na análise substancial do trabalho. Considere que "uma boa revisão é aquela que

31 VASCONCELLOS. Editorial: a função do periódico científico e do editor para a produção do conhecimento no Direito e nas ciências criminais, p. 11.

32 FERREIRA; PINTO; BELFORT. O que é uma boa revisão de artigo em Administração? p. 94-95; HOLLAND. Being a Journal Reviewer, p. 182; HEDDLE; NESS. Reviewing manuscripts, p. 2265-2266.

33 PLATA-CAVIDES; CÓRDOBA-SALGADO; TRZESNIAK. Dictámenes en Revistas Científicas, p. 51 (tradução livre). Assim também: SPYNS; VIDAL. Scientific Peer Reviewing, p. 24.

34 FERREIRA; PINTO; BELFORT. O que é uma boa revisão de artigo em Administração? p. 93-94. 
propõe sugestões de melhoria, apresenta questionamentos substanciais sobre a contribuição teórica, aponta possíveis lacunas, e indica eventual literatura que seja desconhecida pelo autor". ${ }^{35}$

d) Tente se colocar no lugar do autor, buscando pensar a partir dos objetivos e limitações que o artigo se propõe a enfrentar, pois uma das maiores críticas ao controle por pares é que muitas vezes "os avaliadores impõem sugestões idealizando os trabalhos que eles mesmos gostariam de ter escrito, em lugar de analisar o artigo submetido em seus próprios méritos”. ${ }^{36} \mathrm{O}$ controle por pares e as críticas expostas pelos avaliadores e pelos editores não pretendem forçar o autor a alterar seu posicionamento ou adotar uma determinação visão, mas, exatamente ao contrário, almejam problematizar as teses sustentadas no artigo para fomentar a reflexão e, assim, reforçar a consistência do próprio trabalho por meio das rodadas de correções. ${ }^{37}$ Por tal motivo, os autores não são obrigados a aceitar todas as sugestões apresentadas, podendo rejeitar o que entenderem abusivo ou inviável, desde que apresentem motivação adequada para tal recusa. Essa temática será especificamente abordada em editorial posterior, em que se analisará as rodadas de correções e a carta-resposta do autor à aprovação condicionada.

e) $\mathrm{O}$ artigo deve ser analisado de modo imparcial, independente e objetivo. $\mathrm{O}$ avaliador não pode considerar aversões pessoais preconcebidas às teses sustentadas no artigo por questões diversas de sua consistência e fundamentação científica. ${ }^{38}$ Isso não impede (ou, ao menos, não deveria) que pesquisadores com posições distintas (por ex. por partirem de premissas diferentes) avaliem um artigo que sustente tese avessa

35 FERREIRA; PINTO; BELFORT. O que é uma boa revisão de artigo em Administração? p. 90.

36 LEBLECICI, The act of reviewing and being a reviewer, p. 271 (tradução livre). Assim também: ARAÚJO. Revisão por pares, p. e33.

37 FERREIRA; PINTO; BELFORT. O que é uma boa revisão de artigo em Administração? p. 96.

38 Conforme dados obtidos em pesquisa empírica realizada por Arthur Bedeian, mais de um terço dos autores afirmam que parte das recomendações apresentadas a seus artigos em controle por pares são baseadas indevidamente em preferências pessoais de avaliadores e editores, caracterizando, portanto, um problema que carece de atenção no estudo do processo editorial científico (BEDEIAN, Arthur. The manuscript review process, p. 333). 
às suas concepções. Contudo, se o revisor, em última análise, realmente considerar que as premissas adotadas pelo autor (ou qualquer outro motivo) inviabilizam a sua avaliação imparcial, deve recusar a avaliação por "conflito de interesses", apresentando tal justificação ao editor.

f) $\mathrm{O}$ avaliador deve resguardar o sigilo dos dados e das informações contidas no artigo analisado, não podendo utilizar ou divulgar tais ideias sem autorização do autor ou a devida referência, após a publicação. ${ }^{39}$ Nesse sentido, em regra, os avaliadores não estão autorizados a compartilhar o manuscrito ou discutir sobre ele com outras pessoas. ${ }^{40} \mathrm{O}$ revisor somente poderá solicitar a ajuda de outro pesquisador se for autorizado pelo editor, de modo que deve entrar em contato previamente e especificar quem atuaria conjuntamente na redação do parecer.

Assentadas tais premissas gerais da atuação do revisor no controle por pares em periódicos científicos, pode-se elencar algumas orientações práticas para facilitar a atuação do avaliador (III). Tais dicas serão elencadas em três tópicos: 1) recebimento e resposta à solicitação de parecer; 2) orientações gerais sobre a avaliação; e, 3) redação do parecer.

1) Recebimento e resposta à solicitação de parecer. Após realizar o controle preliminar e atestar a viabilidade inicial da submissão, o editor irá selecionar dois ou mais avaliadores (em regra, três) na lista do Corpo de Avaliadores da RBDPP para solicitar a revisão do artigo. Então, um e-mail é enviado, contendo instruções preliminares, o prazo fixado para conclusão e dados básicos do artigo (título e resumo). Primeiramente, o avaliador deverá comunicar se aceita ou não a solicitação, o quanto antes, para somente depois ter acesso ao arquivo do artigo e ao formulário de avaliação. Nesse momento de contato inicial, o parecerista deve considerar três questões para decidir se aceita tal responsabilidade - interesse, imparcialidade e disponibilidade: ${ }^{41}$

a) Compatibilidade temática (interesse). A ideia básica do controle por pares é que um especialista na temática específica irá avaliar

39 WINCK; WEDZICHA; FONSECA; AZEVEDO. To publish or perish, p. 98; HEDDLE; NESS. Reviewing manuscripts, p. 2266; HAMES. Peer Review and Manuscript Management in Scientific Journals, p. 147.

${ }^{40}$ LUSHER. Peer Review Process, Editorial Decisions, and Manuscript Resubmission, p. 567.

${ }^{41}$ WINCK; WEDZICHA; FONSECA; AZEVEDO. To publish or perish, p. 98. 
o conhecimento produzido por outro especialista. Portanto, a escolha do editor deve se pautar, fundamentalmente, por tal critério, buscando avaliadores que tenham conhecimento destacado sobre a questão abordada, o que se verificará pelas palavras-chave indicadas no cadastro do pesquisador no sistema da RBDPP ou em outros mecanismos, como Lattes ou Orcid, por meio das produções anteriores do avaliador. ${ }^{42}$ Ao receber a solicitação, deve-se verificar se o tema é compatível com suas pesquisas e conhecimentos prévios.

b) Ausência de conflito de interesses (imparcialidade)..$^{43} \mathrm{~A}$ avaliação do artigo deve se dar de um modo imparcial e sem favorecimentos ou prejuízos. O parecerista deve verificar se há algum conflito de interesses que possa macular a análise do artigo exclusivamente por critérios científicos. Por exemplo, se presumir que o texto é oriundo de algum colega de Universidade, familiar ou amigo, impõe-se a recusa da solicitação. Em caso de dúvida, deve-se entrar em contato com o editor. ${ }^{44}$

c) Disponibilidade. Conforme pesquisas empíricas, a realização de uma avaliação consistente toma, em média, 3 a 5 horas..$^{45}$ Certamente, trata-se de questão muito variável, conforme o tamanho do artigo, a experiência do avaliador, a complexidade da temática, etc. Assim, ao receber a solicitação de parecer, verifique na sua agenda a disponibilidade de, pelo menos, um turno de seu dia (3-5 horas) para esta tarefa em data anterior ao prazo fixado (e já anote o compromisso para evitar esquecimento). Não há qualquer problema em se recusar uma solicitação (embora negativas reiteradas possam ocasionar o descredenciamento),

42 Em pesquisa com editores, afirmou-se que "determinar qual é o revisor adequado para cada revisão" é a tarefa mais difícil na sua atuação (SILVA; MOREIRO-GONZALEZ; MUELLER. M. A revisão por pares a partir da percepção dos editores, p. 141). De modo semelhante: HAMES. Peer Review and Manuscript Management in Scientific Journals, p. 43.

43 HAMES. Peer Review and Manuscript Management in Scientific Journals, p. 164-169.

44 HEDDLE; NESS. Reviewing manuscripts, p. 2265.

45 Expondo, por meio de pesquisa empírica, que a média é 5 horas: WARE. Peer review survey 2015 , p. 34 . Por outro lado, afirmando que avaliadores com experiência tendem a demorar de 2 a 3 horas: ROSENFELD. How to review journal manuscripts, p. 473; SNELL; SPENCER. Reviewers' perceptions of peer review process for a medical journal, p. 92; LEE. How to be a great reviewer, p. 158. 
mas se você aceitá-la, por favor, cumpra com o prometido, pois o não retorno do parecer após o transcurso de todo o prazo prejudica em muito o andamento do processo editorial.

Após o aceite da solicitação, o revisor poderá obter o arquivo anônimo do artigo e ter acesso ao formulário de avaliação, o qual poderá ser analisado antes da leitura do trabalho para já orientar o contato do avaliador com o artigo. Assim, deve-se indicar algumas orientações gerais sobre a avaliação (2):

a) Primeiro contato e visão geral do artigo. Para iniciar a avaliação do artigo, sugere-se que, no primeiro contato, o parecerista busque uma compreensão geral do artigo, analisando seu título, resumo, sumário (títulos dos tópicos), a consistência das notas de rodapé e o referencial teórico utilizado. ${ }^{46}$ Assim, o revisor poderá ter uma visão inicial das premissas e objetivos do autor, além de perceber o problema e a tese que, idealmente, devem ser claros no manuscrito. Uma crítica que é reproduzida por parte dos avaliadores brasileiros e deve ser fragilizada é que o trabalho não poderia antecipar sua tese (e conclusões) na introdução e no resumo: bem pelo contrário, é fundamental que isso esteja precisamente exposto ao leitor desde o primeiro contato.

Após tal exame superficial, pode-se ler rapidamente a introdução e a conclusão do texto, de modo a consolidar e confirmar a compreensão sobre os objetivos, problema e tese sustentados pelo autor. Nesse momento, o avaliador já deve poder perceber, preliminarmente, se a temática do artigo é atual e compatível com o periódico e se há uma potencial contribuição inovadora e original ao conhecimento da área. É interessante tomar nota dessa ideia inicial formada pelo revisor com o primeiro contato, de modo a, após a leitura integral, verificar se as exposições do autor no título, resumo e introdução estão adequadas ao conteúdo do trabalho.

b) Avalie o artigo integralmente, ainda que tenha verificado logo ao iniciar um problema que, na sua visão, imponha a recomendação de rejeição. ${ }^{47}$ Não pare de ler o texto ao encontrar uma deficiência grave.

\footnotetext{
46 FERREIRA. Como rever um artigo, p. 4.

47 ROBERTS; COVERDALE; EDENHARDER; LOUIE. How to Review a Manuscript, p 84; ARAÚJO. Revisão por pares, p. e34.
} 
Critique e aponte sugestões de correções do modo mais completo que puder, mesmo nos casos de rejeição, pois, além de possibilitar o aprimoramento do artigo para eventual submissão a outro periódico, a decisão sobre aprovação/rejeição é do editor, que, considerando os pareceres dos avaliadores, também analisa o artigo e valora a situação ampla do fluxo editorial da revista. ${ }^{48}$

c) Ainda que o trabalho esteja muito bom, se esforce para problematizar as teses sustentadas e buscar o aprimoramento do texto: nenhum artigo é perfeito, de modo que avaliações que somente expressam a excelência do trabalho em poucas linhas são inconsistentes, não contribuindo em sua total potencialidade para o processo editorial. ${ }^{49}$ Sempre há alguma sugestão, problematização ou indicação de bibliografia complementar a ser feita.

d) Depois de ler o artigo integralmente, revise o título, as palavras-chaves e o resumo, verificando se realmente descrevem o trabalho com precisão. Tais elementos precisam ser autoexplicativos, expondo claramente a temática, o problema e a tese sustentada. ${ }^{50}$

Após (ou durante) a análise do artigo, deve-se redigir o parecer, fundamentalmente a partir dos critérios e questionamentos expostos no formulário de avaliação da RBDPP. Para isso, (3) algumas orientações podem ser indicadas com o objetivo de fomentar revisões de excelência:

a) Comece o parecer com um breve resumo da(s) ideia(s) principal(is) do artigo avaliado e com os pontos positivos do trabalho (sempre há algum mérito, por menor que ele seja valorado pelo revisor). ${ }^{51}$ Isso é fundamental para demonstrar que o avaliador efetivamente compreendeu as questões centrais do texto e para romper uma preconcebida aversão do autor (futuro leitor do parecer): as críticas serão muito

48 FERREIRA; PINTO; BELFORT. O que é uma boa revisão de artigo em Administração? p. 95-96.

49 ROSENFELD. How to review journal manuscripts, p. 484; PROVENZALE; STANLEY. A systematic guide to reviewing a manuscript, p. 851.

50 ARAÚJO. Revisão por pares, p. e33.

51 "Eu sempre penso que a primeira responsabilidade do avaliador é ter uma boa compreensão geral dos objetivos fundamentais do artigo e comunicar essa percepção no início do parecer" (LEBLECICI, The act of reviewing and being a reviewer, p. 270) (tradução livre). De modo semelhante: HOPPIN JR. How I review an original scientific article, p. 1022. 
melhor recebidas assim. Afirma-se que "o parecer mais útil é aquele que expõe os pontos fortes do artigo e identifica consistentemente suas limitações para aprimoramento posterior". ${ }^{2}$ Apontar os pontos positivos $e$ as contribuições que o artigo proporciona (ou pode proporcionar) é tão importante quanto especificar seus problemas e deficiências, pois a função do avaliador não é buscar indistintamente motivos para a rejeição. ${ }^{53}$

b) Adote um tom respeitoso e não agressivo. ${ }^{54}$ Inevitavelmente, receber críticas fortes a um trabalho que demandou tempo e esforço causa uma resistência inicial no autor, que deverá se esforçar para receber positivamente as problematizações. Portanto, ainda que o revisor não esteja mal-intencionado e não tenha qualquer pretensão de ofender, isso é algo que pode ocorrer involuntariamente no panorama do processo editorial. Por isso, além de obviamente se predispor a adotar um tom respeitoso e não agressivo, releia todo o parecer antes de finalizar, buscando suavizar o texto. Por óbvio, não se propõe aqui que o avaliador exclua/atenue críticas ou não problematize profundamente o trabalho, pois, como exposto neste editorial, boas avaliações são rígidas, consistentes e construtivas. Contudo, pequenas alterações no modo como a crítica é exposta podem diminuir o impacto negativo inicial do parecer ao autor. Por exemplo, tente dirigir as críticas não diretamente ao autor, mas ao texto, escrevendo "o artigo sustenta... e isso pode ser questionado porque...", em lugar de "o autor defende... e isso é insustentável". ${ }^{55}$

c) Evite críticas genéricas e vagas. Sempre tente apontar a página e transcrever o trecho específico do texto que está sendo criticado; indique referências bibliográficas fundamentais na temática que não foram utilizadas e não somente afirme que "a bibliografia é insuficiente". Conforme Spyns e Vidal, "boas críticas identificam claramente o problema (também por indicar precisamente o trecho do trabalho avaliado) e sugerem opções para sua redução ou correção". ${ }^{56}$ Para tanto, é

52 ROBERTS; COVERDALE; EDENHARDER; LOUIE. How to Review a Manuscript, p. 82 (tradução livre).

53 WINCK; WEDZICHA; FONSECA; AZEVEDO. To publish or perish, p. 98; SPYNS; VIDAL. Scientific Peer Reviewing, p. 36.

54 HOPPIN JR. How I review an original scientific article, p. 1019.

55 PROVENZALE; STANLEY. A systematic guide to reviewing a manuscript, p. 849.

56 SPYNS; VIDAL. Scientific Peer Reviewing, p. 43 (tradução livre). 
recomendável estruturar as críticas, problematizações e sugestões em itens numericamente (ponto-por-ponto), separando cada ideia de um modo claro, de modo a facilitar a análise pelo editor e a posterior resposta do autor. ${ }^{57}$

d) Sempre que possível fundamente suas críticas e problematizações em referências doutrinárias, ${ }^{58}$ de modo a contribuir com a indicação de bibliografia complementar ao autor em diversos sentidos, favoráveis ou contrárias à tese sustentada.

e) Idealmente, além de preencher o formulário (modelo) de avaliação, envie também o próprio arquivo do artigo com as suas marcações, preferencialmente com a utilização da ferramenta de revisão do WORD. É possível fazer isso pelo próprio sistema da revista, conforme as instruções contidas na página com os dados da avaliação.

Por fim, vale ressaltar que, como medida de valorização do trabalho do avaliador, a RBDPP encaminha a todos os envolvidos (sem identificação de pareceristas e autores) o e-mail da comunicação da decisão preliminar sobre o artigo submetido, juntamente com os pareceres e a decisão editorial. ${ }^{59}$ Trata-se de condição fundamental para a transparência do processo editorial e também incentivo para o aprimoramento das avaliações, de modo que se sugere aos revisores sempre analisar os demais pareceres e verificar o impacto dos seus apontamentos na decisão editorial para aprimorar constantemente a sua atuação como revisor. ${ }^{60}$ Contudo, não receba negativamente o fato de o editor eventualmente não adotar na totalidade a sua recomendação: a decisão editorial é orientada pelos pareceres dos avaliadores, mas não só, pois, além de o próprio edi-

57 WINCK; WEDZICHA; FONSECA; AZEVEDO. To publish or perish, p. 98; ROSENFELD. How to review journal manuscripts, p. 476.

58 WINCK; WEDZICHA; FONSECA; AZEVEDO. To publish or perish, p. 98.

59 Em pesquisa empírica, afirmou-se que 90\% dos avaliadores entrevistados pensam que é ou seria muito útil receber os comentários dos demais avaliadores do artigo para aprimorar a própria atuação como revisor (SNELL; SPENCER. Reviewers' perceptions of peer review process for a medical journal, p. 94).

60 Afirmando que a análise dos demais pareceres e da decisão editorial é fundamental para o desenvolvimento da atuação do avaliador: LEBLECICI, The act of reviewing and being a reviewer, p. 270; HAMES. Peer Review and Manuscript Management in Scientific Journals, p. 140. 
tor revisar o artigo, são considerados aspectos amplos do processo editorial do periódico, como a quantidade e qualidade dos artigos em avaliação no momento e a situação da preparação da próxima publicação. ${ }^{61}$

Diante do exposto neste editorial, reitera-se a conclusão de que a consolidação dos periódicos científicos no Direito como meios adequados para publicação de conhecimento consistente é fundamental para o desenvolvimento da produção científica na área. ${ }^{62}$ Nesse sentido, a excelência dos periódicos passa necessariamente pela qualificação e valorização dos envolvidos no processo editorial: editores, revisores e autores.

\section{REFERÊNCIAS}

ALI, P.; WATSON, R. Peer review and the publication process. Nursing Open, vol. 3, n. 4, p. 193-202, 2016. https://doi.org/10.1002/nop2.51

ARAÚJO, Claudio G. S. Revisão por pares: um processo científico em constante aprimoramento. Arquivos Brasileiros de Cardiologia, São Paulo, v. 98, n. 2, p. e32-e35, fev. 2012. https://doi.org/10.1590/S0066-782X2012000200017

BAILEY, Charles; HAIR, Joe; HERMANSON, Dana; CRITTENDEN, Victoria. Marketing Academics' Perceptions of the Peer Review Process. Marketing Education Review, vol. 22, n. 3, p. 263-278, 2012. https://doi.org/10.2753/ MER1052-8008220306

BEDEIAN, Arthur. The manuscript review process: the proper roles of authors, referees, and editors. Journal of Management Inquiry, vol. 12, n. 4, p. 331-338, 2003. https://doi.org/10.1177/1056492603258974

BIGGS, Mary. The impact of peer review on intellectual freedom. Library Trends, vol. 39, n. 1-2, p. 145-167, 1990.

CAMPANARIO, Juan Miguel. Have Referees Rejected Some of the MostCited Articles of All Times? Journal of the American Society for Information Science, vol. 47, n. 4, p. 302-310, 1996. https://doi.org/10.1002/(SICI)10974571(199604)47:4<302::AID-ASI6>3.0.CO;2-0

${ }^{61}$ ROSENFELD. How to review journal manuscripts, p. 484; LEE. How to be a great reviewer, p. 159.

${ }^{62}$ “(...) a publicação em periódicos poderia levar a uma melhoria na qualidade científica do conhecimento produzido na área do Direito, uma vez que os periódicos passam por processos muito mais rigorosos de avaliação e controle de cientificidade" (SILVEIRA; SANCHES. Periódicos na área do Direito, p. 158). 
CAMPANARIO, Juan Miguel. Peer review for journals as it stands today part 1. Science Communication, vol. 19, n. 3, p. 181-211, 1998. https://doi. org/10.1177/1075547098019003002

CAPPARELLI, Bruna. Editorial: Sobre a exigência de um método de escrita de artigos científicos e de estudo do Direito Processual Penal. Revista Brasileira de Direito Processual Penal, Porto Alegre, vol. 3, n. 1, p. 19-27, jan./abr. 2017. https://doi.org/10.22197/rbdpp.v3i1.37

COMMONER, Barry. Peering at Peer Review. Hospital Practice, vol. 13, n. 11, p. 25-29, 1978. DOI: https://doi.org/10.1080/21548331.1978.11707427

CORONEL, Ruben; OPTHOF, Tobias. The role of the reviewer in editorial decision-making. Cardiovascular Research, vol. 43, n. 2, p. 261-264, 1999. https://doi.org/10.1016/S0008-6363(99)00177-7

DESLANDES, Suely F.; SILVA, Antônio A. M. Revisão por pares: crise de demanda ou mudança de valores?. Cadernos de Saúde Pública, Rio de Janeiro, v. 29, n. 3, p. 421-423, mar. 2013. https://doi.org/10.1590/S0102-311X2013000300001

FERREIRA, Manuel A. Como rever um artigo: o papel do revisor e um roteiro para novos revisores (comentário editorial). Revista Ibero-Americana de Estratégia, São Paulo, v. 13, n. 2, p. 1-9, abr./jun. 2014. https://doi.org/10.5585/ riae.v13i2.2110

FERREIRA, Manuel A.; CANELA, Renata; PINTO, Cláudia F. O processo editorial nos periódicos e sugestões para a publicação. Revista de Gestão e Secretariado, São Paulo, v. 5, n. 2, p. 01-22, mai./ago. 2014. https://doi.org/10.7769/gesec. v5i2.307

FERREIRA, Manuel A.; PINTO, Cláudia F.; BELFORT, Ana C. O que é uma boa revisão de artigo em Administração? Revista Eletrônica de Estratégia \& Negócios, v. 9, n. 2, p. 87-105, mai./ago. 2016. https://doi.org/10.19177/reen. v9e2201686-104

FISKE, Donald; FOGG, Louis. But the reviewers are making different criticisms of my paper! Diversity and uniqueness in reviewer comments. American Psychologist, vol. 45, n. 5, p. 591-598, 1990. https://doi.org/10.1037/0003-066x.45.5.591

GOODMAN, Steven; BERLIN Jesse; FLETCHER Suzanne; FLETCHER Robert. Manuscript quality before and after peer review and editing at Annals of Internal Medicine. Annals of Internal Medicine, vol. 121, p. 11-21, 1994. https:// doi.org/10.7326/0003-4819-121-1-199407010-00003

HAACK, Susan. La evaluación por pares y la publicación: lecciones para abogados. DOXA - Cuadernos de Filosofía del Derecho, vol. 38, p. 15-40, 2015. https://doi.org/10.14198/DOXA2015.38.01 
HAMES, Irene. Peer Review and Manuscript Management in Scientific Journals. Malden: Blackwell, 2007. https://doi.org/10.1002/9780470750803

HEDDLE, Nancy; NESS, Paul. Reviewing manuscripts: tips and responsibilities. Transfusion, vol. 49, p. 2265-2268, 2009. https://doi.org/10.1111/j.15372995.2009.02390.x

HOLLAND, Karen. Being a Journal Reviewer: Good Practice in Reviewing. In: HOLLAND, Karen; WATSON, Roger (eds.). Writing for Publication in Nursing and Healthcare. Hoboken: Wiley-Blackwell, 2012.

HOPPIN JR., Frederic. How I review an original scientific article. American Journal of Respiratory and Critical Care Medicine, vol. 166, n. 8, p. 1019-1023, 2002. https://doi.org/10.1164/rccm.200204-324oe

JENAL, S.; VITURI, D.; EZAÍAS, G.; SILVA, L.; CALIRI, M. O processo de revisão por pares: uma revisão integrativa de literatura. Acta paulista de enfermagem, São Paulo, v. 25, n. 5, p. 802-808, 2012 https://doi.org/10.1590/S010321002012000500024

LABAND, David; PIETTE, Michael. A citation analysis of the impact of blinded peer review. JAMA, vol. 272, n. 2, p. 147-149, 1994. https://doi.org/10.1001/ jama.1994.03520020073020

LARSON, Bradley; CHUNG, Kevin. A systematic review of peer review for scientific manuscripts. HAND, vol. 7, p. 37-44, 2012. https://doi.org/10.1007/ s11552-012-9392-6

LEBLECICI, Huseyin. The act of reviewing and being a reviewer. In: FROST; TAYLOR (Eds.). Rhythms of academic life. Thousand Oaks: Sage, 1996. p. 269274. https://doi.org/10.4135/9781452231570.n27

LEE, Samuel S. How to be a great reviewer: an editor's view. Liver International, vol. 28, n. 2, p. 158-159, 2008. https://doi.org/10.1111/j.1478-3231.2007.01656.x

LEOPOLD, Seth. Editorial: Peer Review and the Editorial Process - A Look Behind the Curtain. Clinical Orthopaedics and Related Research, vol. 473, n. 1, p. 1-3, 2015. https://doi.org/10.1007/s11999-014-4031-x

LEUNG, Daniel; LAW, Rob; KUCUKUSTA, Deniz; GUILLET, Basak D. How to review journal manuscripts: A lesson learnt from the world's excellent reviewers. Tourism Management Perspectives, vol. 10, p. 46-56, 2014. https:// doi.org/10.1016/j.tmp.2014.01.003

LOCK, Stephen. A difficult balance. Editorial peer review in medicine. London: The Nuffield Provincial Hospitals Trust, 1985.

LUSHER, Audrey. Peer Review Process, Editorial Decisions, and Manuscript Resubmission: a reference for novice researchers. The Journal of the American 
Osteopathic Association, vol. 115, n. 9, p. 566-569, 2015. https://doi.org/10.7556/ jaoa.2015.114

MARTÍNEZ, Gladys. La revisión por pares y la selección de artículos para publicación. Revista Colombiana de Psicología, vol. 21, n. 1, p. 27-35, jan.-jun. 2012.

PLATA-CAVIDES, Tatiana; CÓRDOBA-SALGADO, Oscar A.; TRZESNIAK, Piotr. Dictámenes en Revistas Científicas: lo que Necesitan los Editores y Autores, lo que Hacen los Evaluadores. Revista Colombiana de Psicología, vol. 21, n. 1, p. 3755, jan.-jun. 2012.

PROVENZALE, James; STANLEY, Robert. A systematic guide to reviewing a manuscript. American Journal of Roentgenology, vol. 185, p. 848-854, 2005. https://doi.org/10.2214/AJR.05.0782

RAJPERT-DE MEYTS, E.; LOSITO, S.; CARRELL, D. Rewarding peer-review work: the Publons initiative. Andrology, vol. 4, p. 985-986, 2016. https://doi. org/10.1111/andr.12301

ROBERTS, Laura; COVERDALE, John; EDENHARDER, Kristin; LOUIE, Alan. How to Review a Manuscript: A "Down-to-Earth" Approach. Academic Psychiatry, vol. 28, n. 2, p. 81-87, 2004. https://doi.org/10.1176/appi.ap.28.2.81 ROSENFELD, Richard. How to review journal manuscripts. OtolaryngologyHead and Neck Surgery, vol. 142, p. 472-486, 2010. https://doi.org/10.1016/j. otohns.2010.02.010

SHATTELL, M.M.; CHINN, P.; THOMAS, S.; COWLING, W. Authors' and editors' perspectives on peer review quality in three scholarly nursing journals. Journal of Nursing Scholarship, vol. 42, n. 1, p. 58-65, 2010. https://doi.org/10.1111/ j.1547-5069.2009.01331.x

SHIGAKI, Helena; PATRUS, Roberto. Revisão por pares e produtivismo acadêmico sob a ótica de avaliadores de artigos de periódicos em Administração. Revista Brasileira de Pós-graduação, Brasília, v.13, n. 31, p. 399-428, mai./ago. 2016. https://doi.org/10.21713/2358-2332.2016.v13.960

SILVA, Cláudio N.; MOREIRO-GONZALEZ, José A; MUELLER, Suzana P. M. A revisão por pares a partir da percepção dos editores: um estudo comparativo em revistas brasileiras, espanholas e mexicanas. Revista Digital de Biblioteconomia e Ciência da Informação, Campinas, SP, v. 14, n. 1, p. 126-143, fev. 2016. http:// dx.doi.org/10.20396/rdbci.v14i1.8640579

SILVEIRA, Vladmir O.; SANCHES, Samyra H. F. N. Periódicos na área do Direito: o desafio da superação da cultura dos livros. Revista da Faculdade de Direito da UERJ, Rio de Janeiro, n. 30, p. 157-172, dez. 2016. http://dx.doi.org/10.12957/ rfd.2016.17961 
SMITH, David R. Will Publons Popularize the Scientific Peer-Review Process? BioScience, vol. 66, n. 4, p. 265-266, 2016. https://doi.org/10.1093/biosci/biw010 SNELL, Linda; SPENCER, John. Reviewers' perceptions of peer review process for a medical journal. Medical Education, vol. 39, p. 90-97, 2005. https://doi. org/10.1111/j.1365-2929.2004.02026.x

SOLOMON, David. The Role of Peer Review for Scholarly Journals in the Information Age. Journal of Electronic Publishing, vol. 1, n. 1, p. 1-5, jan. 2007. https://doi.org/10.3998/3336451.0010.107

SPYNS, Peter; VIDAL, María-Esther. Scientific Peer Reviewing: Pratical hints and best practices. Heidelberg: Springer, 2015. https://doi.org/10.1007/9783-319-25084-7

TRZESNIAK, Piotr. A estrutura editorial de um periódico científico. In: SABADINI; SAMPAIO; KOLLER (Orgs.). Publicar em psicologia: um enfoque para a revista científica. São Paulo: ABECiPsi, 2009. https://doi.org/10.13140/ RG.2.1.2810.6965

VASCONCELLOS, Vinicius G. Editorial: a função do periódico científico e do editor para a produção do conhecimento no Direito e nas ciências criminais. Revista Brasileira de Direito Processual Penal, Porto Alegre, vol. 3, n. 1, p. 9-17, jan./abr. 2017. https://doi.org/10.22197/rbdpp.v3i1.34

WARE, Mark. Peer review: benefits, perceptions and alternatives. London: Publishing Research Consortium, 2009. Disponível em: <http://publishingresearchconsortium. com/index.php/prc-documents/prc-research-projects/35-prc-summary-4-warefinal-1/file>. Acesso em: 28 mar. 2017.

WARE, Mark. Peer review survey 2015. London: Publishing Research Consortium, 2016. Disponível em: <http://publishingresearchconsortium.com/index.php/ prc-documents/prc-research-projects/57-prc-peer-review-survey-2015/file>. Acesso em: 28 mar. 2017.

WERLANG, Elisabete. Revisão por pares: um estudo da gestão de avaliadores nas revistas científicas brasileiras. 2013. Dissertação (Mestrado em Ciência da Informação) - Centro de Ciências da Educação, Universidade Federal de Santa Catarina. https://doi.org/10.5007/31452

WICHERTS Jelte M. Peer Review Quality and Transparency of the Peer-Review Process in Open Access and Subscription Journals. PLOS ONE, vol. 11, n. 1, p. 1-19, 2016. https://doi.org/10.1371/journal.pone.0147913

WINCK, J.C.; WEDZICHA, J.A.; FONSECA, J.A.; AZEVEDO, L.F. To publish or perish: how to review a manuscript. Revista Portuguesa de Pneumología, vol. 17, n. 2, p. 96-103, 2011. https://doi.org/10.1016/s2173-5115(11)70022-7 


\section{Sugestões de Sites, Documentos e Vídeos}

http://publicationethics.org/files/u7140/Peer review guidelines.pdf https://publons.com/community/academy/

https://authorservices.wiley.com/Reviewers/journal-reviewers/how-toperform-a-peer-review/index.html

https://www.publishingcampus.elsevier.com/pages/69/Colleges/College-ofSkills-Training/Peer-review.html

https://www.youtube.com/channel/UCE5uQwLX5wkkJvtnjOFK-Hw https://peerreviewweek.wordpress.com

\section{COMO CITAR ESTE EDITORIAL:}

VASCONCELLOS, Vinicius G. Editorial: Controle por pares e a função do revisor - premissas e orientações para uma avaliação consistente. Revista Brasileira de Direito Processual Penal, Porto Alegre, vol. 3, n. 2, p. 437-458, mai./ago. 2017. https://doi.org/10.22197/rbdpp.v3i2.70 